\title{
COVID Bedfellows: Combining Clinical Skills and Anatomy Laboratories in the DVM Curriculum
}

\author{
Erin D. Malone, Abby L. Brown', Susan J. Spence ${ }^{2}$ \\ Department of Veterinary Population Medicine, University of Minnesota College of Veterinary Medicine, Saint Paul, MN, USA, ${ }^{1}$ Department of Veterinary and \\ Biomedical Sciences, University of Minnesota College of Veterinary Medicine, Saint Paul, MN, USA, ${ }^{2}$ Department of Veterinary Clinical Sciences, University of \\ Minnesota College of Veterinary Medicine, Saint Paul, MN, USA
}

\section{Abstract}

Background: Prior to the fall of 2020, clinical skills and anatomy were considered separate courses with minimal overlap other than being offered to the same cohort of students. We had included clinical skills practice in anatomy labs on an intermittent and variable basis. Many of the skills were included only if time and faculty or staff availability permitted. The coronavirus disease 2019 (COVID19) pandemic made us think differently, particularly as the anatomy space was one of our largest rooms. Methods: Anatomy and clinical skills shared space and content during the 2020-2021 school year in an effort to meet physical distancing and other biosafety requirements required by the state and University. Results: Students were maintained in a single space and were engaged for a longer period of time, making their commute and biosafety steps worthwhile while simultaneously opening up other spaces for distanced teaching needs. The goal of one skill/day for motor skill learning was maintained despite a challenging laboratory teaching schedule. Certified veterinary technicians taught both the anatomy and the skills portion, showcasing the value of their training and freeing faculty for other responsibilities. Conclusion: This teaching and learning combination led to an enhanced understanding of procedurerelated anatomy; direct anatomy application to veterinary work; opportunities for recall, transfer, and supervised practice; and optimized staff and space utilization.

Keywords: Anatomy, clinical skills, integration, psychomotor skills, veterinary curriculum

A bedfellow is not only someone who shares a space but someone who associates with another to give assistance or support. ${ }^{[1]}$ When physical distancing, ventilation needs, and cleaning requirements changed our teaching paradigm in the fall of $2020,{ }^{[2]}$ our clinical skills and anatomy courses became bedfellows in the full sense of the word. We moved all of our first-year clinical skills laboratory sessions into the anatomy space and expanded the laboratory period by an hour, effectively sequestering each group of students in one space during their weekly visit into the building. This partnership between anatomy and clinical skills rapidly formed into a workable and logical framework for the future. We will continue the cohabitation as it has surpassed our expectations on many fronts. The key advantages are described below.

\begin{tabular}{|l|l|}
\hline \multicolumn{2}{|c|}{ Access this article online } \\
\hline Quick Response Code: & Website: \\
\hline & www.ehpjournal.com \\
\hline & \\
\hline
\end{tabular}

\section{Accurate Practice of Motor Skills}

Prior to the fall of 2020, we had included clinical skills practice in anatomy labs on an intermittent and variable basis. Many of the skills were included only if time and faculty or staff availability permitted. These practice sessions tended to be the first thing to be dropped if students were worried about overwhelming content or upcoming exams. One exception with staying power was instrument handling. By the time students reached the surgery courses, many had developed poor instrument

\section{Address for correspondence: Dr. Erin D. Malone, Department of Veterinary Population Medicine University of Minnesota College of Veterinary Medicine, Saint Paul, MN 55108. E-mail: malon001@umn.edu}

This is an open access journal, and articles are distributed under the terms of the Creative Commons Attribution-NonCommercial-ShareAlike 4.0 License, which allows others to remix, tweak, and build upon the work non-commercially, as long as appropriate credit is given and the new creations are licensed under the identical terms.

For reprints contact: reprints@medknow.com

How to cite this article: Malone ED, Brown AL, Spence SJ. COVID bedfellows: Combining clinical skills and anatomy laboratories in the DVM curriculum. Educ Health Prof 2021;4:70-3.

Submission: 16-02-2021

Revision: 21-05-2021

Acceptance: 02-06-2021 Web Publication: 15-09-2021 
handling skills, particularly for ring instruments (the notorious "death grip") and thumb forceps (held over the top). These habits seemed to develop due to the challenges of removing tissue layers in anatomy, where force was often used to replace finesse. As motor skills are well retained, ${ }^{[3-5]}$ these engrained practices were hard to correct.

To correct the downstream issues, we introduced instrument handling before the first anatomy dissection laboratory starting in 2014. Since that time, over $90 \%$ of students have passed their first-year spring clinical skills assessments on instrument handling and knot tying in their first attempt. Prior to 2014, instrument handling was taught but not well assessed, and knot tying was one of the most common causes for exam retakes (approximately one-third of students needed to repeat either knot tying or open gloving). Overall, students seem much more at ease and competent with the related skills since the 2014 integration. One student reported that other students at the spring veterinary student symposium were impressed by their advanced skills at instrument handling. With the laboratory staff and faculty trained in on what to monitor, most students now master proper instrument handing before the end of fall semester. Blade handling safety is also improved.

\section{Application to Enhance Engagement}

When we moved clinical skills into the anatomy space, we also deliberately connected topics to related anatomy modules. The practice was not entirely new. Prior to 2020, clinical skills had partnered with the anatomy teaching team to create cadaveric prosections of the target muscle groups for the intramuscular (IM) injection laboratory topic. Students would happily place their index finger on the dorsal spinous processes of the lumbar spine and their thumb just cranial to the wing of the ilium forming an "L" with their left hand and inject into the right epaxial muscle group. However, they struggled to properly inject the left epaxial muscle group and also struggled with avoiding the sciatic nerve when asked to inject into the hindquarters. Having the prosections to visually demonstrate muscle locations relative to the sciatic nerve helped students identify the value of hand positioning and how to adjust based on the animal's position. Instructors also recommend that students adjust the "patient" to the laterality (or sternal recumbency) that works for them. In the fall of 2020, the clinical skills IM injection laboratory topic was tied in with the dissection of the hind limb in anatomy, since many IM injection sites are associated with the hind limb. Prosected animals were still used for demonstration during the clinical skills session. Additionally, those muscle groups/injection sites and location of the sciatic nerve were further reinforced when the students completed their anatomy dissection of the hind limb.

In 2020, subcutaneous (SQ) injections were connected to the first anatomy dissection laboratory session. That session focused on SQ tissues, fascial planes, and forelimb anatomy. Performing SQ injections prior to the dissection made that anatomy directly relevant. Handwashing (for biosecurity) was another immediate and early skill connection. Instead of setting up Glo Germ tents in the clinical skills space, we moved the whole module into the anatomy laboratory so that students could see how effective their handwashing technique was prior to putting on gloves to participate in the laboratory session (similar to a nonsterile clinical setting). This activity carried particular significance as part of the enhanced biosecurity needs. The clinical skills venipuncture/intravenous catheter session was completed after the anatomic dissection of the limbs and neck to correlate the locations of superficial veins commonly used for venipuncture with the skills practice. In the spring semester of 2021, the clinical skills cohabitation with anatomy continued and the bandaging topic was integrated into the large animal (equine and bovine) thoracic limb dissection lab session, utilizing the large animal skeletons and small animal leg models.

A few modules were less directly connected but continued in the anatomy space to optimize space use during the coronavirus disease (COVID) times. These are included in Table 1.

\section{Table 1: Linked clinical skills and anatomy laboratories}

\begin{tabular}{ll}
\hline Clinical skill & Anatomy module \\
\hline Instrument handling (thumb forceps, scalpels, scissors) & Occurred before the first anatomy laboratory \\
Handwashing & Anatomy introduction \\
SQ injections: syringe handling, vaccines, microchipping & Subcutaneous planes and thoracic limb (SA) \\
IM injections: syringe handling, medications & Hind limb (SA) \\
IV stick: venipuncture, IV catheter placement, vacutainers & Neck anatomy, external regions (SA) \\
Mini-isolation protocols & Internal thorax (SA) \\
Scrub and open glove & Abdomen (SA) \\
Instrument ties, simple interrupted sutures & Head (SA) \\
Limb bandaging & Thoracic limb (LA) \\
Laboratory testing (hematocrit, total protein, etc.) & Pelvic limb (LA) \\
Progress testing and refresher practice & Body cavities, pelvis, head (LA) \\
\hline
\end{tabular}

IM = intramuscular, IV = intravenous, LA = large animal, SA = small animal, $\mathrm{SQ}=$ subcutaneous

Clinical skills laboratories include both small animal and large animal variants in each session when applicable. Anatomy laboratories are species based with SA in the fall and LA in the spring 


\section{One Skill per Day for Retention}

Motor skills are processed differently than new knowledge. Only one new motor skill is retained at a time. Sleep consolidates and enhances the skill. If a second skill is introduced within a 6-h window, only the second skill is retained ${ }^{[5-8]}$ By distributing the skills to other laboratories that are not focused on motor skills, we hoped to optimize laboratory time and skills retention. While anatomy does teach dissection and enables practice of surgical instrument handling, most of the content is knowledge based, thus less likely to interfere with motor skill retention.

An additional benefit of the partnership has been expanding the large animal content in the first-year curriculum. The fall anatomy course is small animal focused, whereas that of spring is large animal focused. As the clinical skill courses use species variety to optimize the one-skill-perday principle, the partnership encourages extension of anatomy learning across species and ties material across both semesters. Vaccines are given by different routes in different species; students learn about cow and goat vaccines in the SQ unit and horse vaccines in the IM unit even though the associated anatomy units are focusing on small animals. Bandaging small and large animal limbs in the spring encourages recall of small animal anatomy even while learning about the large animal thoracic limb.

\section{Optimized Laboratory Staffing}

Prior to the 2020-2021 school year, a typical first-year clinical skills laboratory was 60-90 min long, which included time for students to move in and out of the laboratory space. Approximately one-fourth of the class ( $\approx 25-30$ students) was in each section of a clinical skills laboratory, and depending upon the session content, one to three veterinarians and one to two staff members were involved in each laboratory. Anatomy laboratories were typically $3 \mathrm{~h}$ in duration; $1 \mathrm{~h}$ took place in the active learning classroom as application activities, followed by $2 \mathrm{~h}$ of dissection. Each anatomy laboratory session included all students $(\approx 100-105)$ and a total of six instructors (three faculty and three staff).

During the 2020-2021 school year, no laboratory session included more than one-sixth of the class (16-18 students in each "pod") and the anatomy active learning sessions were conducted via Zoom. In the fall, a $4 \mathrm{~h}$ time slot was reserved for the combination of anatomy dissection labs and clinical skills training (introductory laboratories). Each "pod" of students completed six combined sessions:

- For three of six sessions, the clinical skills training was completed during the first $60-70 \mathrm{~min}$, followed by anatomy dissection time.

- For one of six sessions, the clinical skills training was completed after dissection, in the last 60-90 min of lab time.

- For two of six sessions, clinical skills and dissection occurred concurrently where small groups of students were called over to complete their clinical skills training $(\approx 10-20 \mathrm{~min} /$ group $)$ and then would return to their dissection.

In the spring, a $3 \mathrm{~h}$ time slot was reserved for the combination of a prosection-based anatomy lab (where students did not dissect but instead rotated through various stations) and clinical skills training (introductory labs and check-ins on skills learned in fall). Each pod of students completed five combined sessions:

- For two of five sessions (per pod of students), the clinical skills training was completed in the first $60 \mathrm{~min}$, followed by rotations through each anatomy station.

- For three of five sessions, the clinical skills check-ins and anatomy stations occurred concurrently where individual students were called over to complete their clinical skills check-in $(\approx 10-15 \mathrm{~min} / \mathrm{student})$ and then would return to their anatomy stations.

Each of the combination sessions (fall and spring) were taught by one to two faculty and two to three staff members.

Combining clinical skills and anatomy in the anatomy laboratory permitted better overall use of physical space, as well as staff and laboratory time. Our anatomy technicians are trained and certified veterinary technicians; these staff members quickly adapted to teaching clinical skills topics alongside the trained clinical skills teaching staff and are well suited to teaching the first-year skills. Furthermore, many of the laboratories tapped into more of the technicians' skill sets, both validating their training and refreshing their skills. A faculty instructor was truly only necessary for the instrument handling (virtual) and suturing laboratories. This added benefit of the union was very welcome with the smaller laboratory groups and more frequent laboratory sessions necessitated by COVID-19 restrictions.

\section{Supervised Practice Time}

Prior to COVID, the clinical skills staff routinely held "check-ins" on previously learned skills. These "checkins" were actually assessments and deliberate practice for challenging skill components. As with knowledge skills, skills assessments provide recall that enhances retention. ${ }^{[3,9]}$ "Check-ins" in prior years lasted 15-20 min/student with multiple waves over $2-3 \mathrm{~h}$ and were staffed by one veterinarian and one staff member. We were unable to have check-ins in the fall of 2020 due to the increased exposure risk with high traffic levels. In the spring of 2021, an additional station for "check-ins" as part of the anatomy/clinical skills combination minimized bottlenecks during anatomy labs, provided a safe avenue for these important assessments, and helped keep students occupied and engaged.

\section{Maintenance of Biosafety Precautions}

During the 2020-2021 school year, the state and the university placed restrictions on the number of people per 
square footage. All used spaces (including restrooms) and high-touch surfaces (including door handles) were cleaned more frequently than usual. As the anatomy laboratory is a large, well-ventilated space, it permitted more physical distancing than did the space used for clinical skills. Students were also able to enter the space through a more direct route, minimizing the number of door handles being touched and limiting shared hallways.

\section{Opportunities Missed and Lessons Learned}

- During the 2020 fall semester, we did not have dog physical examination laboratories. Those were pushed to spring semester in the hope that physical distancing would be safer and more dogs would be in the building as we use staff pets for the laboratories. We will likely shift the mini-isolation unit out of the combined program and have students practice physical examinations during the internal thorax unit. Since these laboratories involve live animals, they would not take place in the anatomy space but would provide more direct application of material.

- Other topics to consider embedding in the anatomy curriculum include practice in body condition scoring, nail trimming, skin suturing, and ear cleaning.

- After the 2020 fall semester, students reported that they preferred to have the clinical skills lesson first, followed by anatomy dissection. This sequence enables the faster groups to leave when dissection is finished and seems to provide fresher energy and a clearer mindset for the skills section while not impairing anatomy dissection.

- Clear, open, and frequent communication among the teaching staff and faculty is critical to the successful partnership of the clinical skills topics and anatomy laboratory sessions. Weekly planning sessions have proven invaluable.

We have gained so much benefit from the combined laboratories that we do not see "separating" them even when permitted post-COVID. The students are learning skills and reinforcing them through anatomy application; the anatomy staff are engaged in a wider variety of activities that encompass more aspects of their training; and other instructors and spaces are freed for additional or alternative learning opportunities. Why go back when living together works so well?

\section{Acknowledgments}

The authors would like to express their deep appreciation for the hard work of the University of Minnesota Teaching
Technician Team-Hannah Gytri, Eileen Kuhlmann, Anna Landherr, and Sally Lightner-and for the support of the new clinical skills "Czar," Dr. Anna Firshman. Without them, this partnership would be dissolved.

\section{Financial support and sponsorship}

Nil.

\section{Conflicts of interest}

There are no conflicts of interest.

\section{Author contribution}

Erin Malone has been managing the University of Minnesota clinical skills program and has been teaching instrument handling since 2014. Abby L. Brown has managed the anatomy laboratories for many years and was instrumental in overseeing technician training and laboratory staffing, as well as coordinating efforts for this partnership. Susan J. Spence coordinates the University of Minnesota small animal clinical skills program, has been involved in the brainstorming and weekly meetings, as well as continues to have a presence in selected first-year laboratories, mostly for the sheer joy of teaching. All were involved in manuscript creation.

\section{RefERENCES}

1. Merriam-Webster.com. Bedfellow [internet]. Springfield, MA. Available from: https://www.merriam-webster.com/thesaurus/ bedfellow. [Last accessed $2021 \mathrm{Feb}$ 15].

2. Centers for Disease Control (CDC): Resources for teachers and staff [internet]. Atlanta, GA, 2020. Available from: https://www.cdc.gov/ coronavirus/2019-ncov/community/schools-childcare/resourcesteachers-staff.html. [Last accessed 2020 Aug 1].

3. Yang CW, Yen ZS, McGowan JE, Chen HC, Chiang WC, Mancini ME, et al. A systematic review of retention of adult advanced life support knowledge and skills in healthcare providers. Resuscitation 2012;83:1055-60.

4. Fisher J, Viscusi R, Ratesic A, Johnstone C, Kelley R, Tegethoff AM, et al. Clinical skills temporal degradation assessment in undergraduate medical education. J Adv Med Educ Prof 2018;6:1-5.

5. Malone E. Challenges \& issues: Evidence-based clinical skills teaching and learning: What do we really know? J Vet Med Educ 2019;46:379-98.

6. Allen SE. Memory stabilization and enhancement following music practice. Psych Music 2012;41:794-803.

7. Appleman ER, Albouy G, Doyon J, Cronin-Golomb A, King BR. Sleep quality influences subsequent motor skill acquisition. Behav Neurosci 2016;130:290-7.

8. Neville KM, Trempe M. Serial practice impairs motor skill consolidation. Exp Brain Res 2017;235:2601-13.

9. Kromon CB, Bohnstedt C, Jensen ML, Ringsted C. The testing effect on skills learning might last 6 months. Adv in Health Sci Educ 2010;15:395-401. 